sion of the RDW-SD in a Cox proportional-hazards model for the development of coronary heart disease did not affect the risk estimate associated with CHIP (hazard ratio, 1.99; 95\% confidence interval, 1.26 to 3.13), after adjustment for RDW-SD, age, sex, hypertension, type 2 diabetes status, smoking history, and levels of high-density lipoprotein cholesterol and total cholesterol. On the basis of this limited sample, we found no evidence that red-cell anisocytosis, as indicated by an elevated RDW-SD, mediates the association between CHIP and atherosclerotic cardiovascular disease.

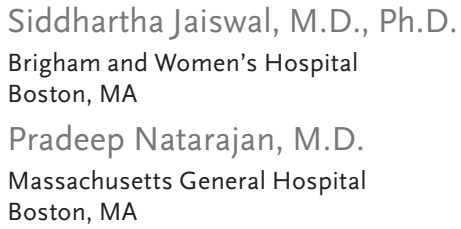

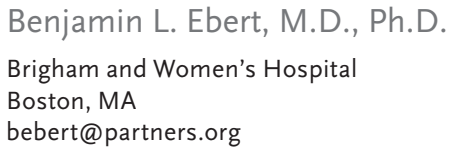

Since publication of their article, the authors report no further potential conflict of interest.

1. Campbell PJ, MacLean C, Beer PA, et al. Correlation of blood counts with vascular complications in essential thrombocythemia: analysis of the prospective PT1 cohort. Blood 2012;120: 1409-11.

2. Carobbio A, Finazzi G, Guerini V, et al. Leukocytosis is a risk factor for thrombosis in essential thrombocythemia: interaction with treatment, standard risk factors, and Jak2 mutation status. Blood 2007;109:2310-3.

3. Landolfi R, Di Gennaro L, Barbui T, et al. Leukocytosis as a major thrombotic risk factor in patients with polycythemia vera. Blood 2007;109:2446-52.

4. Jaiswal S, Fontanillas P, Flannick J, et al. Age-related clonal hematopoiesis associated with adverse outcomes. N Engl J Med 2014;371:2488-98.

DOI: 10.1056/NEJMc1710381

\title{
Itraconazole or Amphotericin B for Talaromycosis
}

TO THE EDITOR: Le et al. (June 15 issue) ${ }^{1}$ report that amphotericin B was superior to itraconazole in the treatment of talaromycosis and that the rates of adverse events were higher with amphotericin B use. However, some issues need to be discussed. The investigators did not report severity of illness or preexisting medical conditions. The treatment of talaromycosis depends on the severity of the infection, because itraconazole is recommended for milder forms of talaromycosis and amphotericin B or voriconazole for more severe forms. ${ }^{2}$ The efficacy of treatment in both study groups can be misleading if, for instance, patients were not adequately treated or were overtreated on the basis of the severity of their infection. The presence of preexisting medical conditions could influence treatment outcomes, because patients with these conditions may be more susceptible to negative outcomes.

\section{Richard A. Giovane, M.D. \\ Paul Manhas, M.D. \\ Katie Gates, M.D. \\ University of Alabama \\ Tuscaloosa, AL \\ richardgiovane357@gmail.com}

No potential conflict of interest relevant to this letter was reported.
1. Le T, Kinh NV, Cuc NTK, et al. A trial of itraconazole or amphotericin B for HIV-associated talaromycosis. N Engl J Med 2017;376:2329-40.

2. Spanakis EK, Aperis G, Mylonakis E. New agents for the treatment of fungal infections: clinical efficacy and gaps in coverage. Clin Infect Dis 2006;43:1060-8.

DOI: $10.1056 / \mathrm{NEJMc1709123}$

TO THE EDITOR: Le et al. compared the efficacy of amphotericin B, administered intravenously, with that of itraconazole, administered orally, in the treatment of talaromycosis and found that amphotericin B was superior. The survival benefit was thought to be associated with greater fungicidal activity of amphotericin $\mathrm{B}$, but the authors did not take into account the highly variable absorption kinetics of itraconazole capsules. Itraconazole is a weakly basic drug, and dissolution of the drug is pH-dependent; thus the drug requires an acidic gastric environment for adequate absorption. ${ }^{1}$ Numerous studies have documented substantial variation in itraconazole exposure owing to the effect of food, interaction with coadministered drugs, and adherence. ${ }^{2}$ Guidelines recommend assessment of the exposure to itraconazole through therapeutic drug monitoring when the drug is used for treatment of systemic 
fungal diseases, ${ }^{3}$ including talaromycosis. ${ }^{4}$ Le et al. do not report on the exposure to itraconazole, but because capsules were used, underdosing of itraconazole might have been a factor in the lower response rate. Information on the exposure to itraconazole would facilitate a better understanding of the differences reported.

Roger J. Brüggemann, Pharm.D., Ph.D.

Frank L. van de Veerdonk, M.D., Ph.D.

Paul E. Verweij, M.D., Ph.D.

Radboud University Medical Center

Nijmegen, the Netherlands

roger.bruggemann@radboudumc.nl

No potential conflict of interest relevant to this letter was reported.

1. Abuhelwa AY, Foster DJ, Mudge S, Hayes D, Upton RN. Population pharmacokinetic modeling of itraconazole and hydroxyitraconazole for oral SUBA-itraconazole and sporanox capsule formulations in healthy subjects in fed and fasted states. Antimicrob Agents Chemother 2015;59:5681-96.

2. Brüggemann RJ, Alffenaar JW, Blijlevens NM, et al. Clinical relevance of the pharmacokinetic interactions of azole antifungal drugs with other coadministered agents. Clin Infect Dis 2009; 48:1441-58.

3. Wheat LJ, Freifeld AG, Kleiman MB, et al. Clinical practice guidelines for the management of patients with histoplasmosis: 2007 update by the Infectious Diseases Society of America. Clin Infect Dis 2007;45:807-25.

4. Panel on Opportunistic Infections in HIV-Infected Adults and Adolescents. Guidelines for the prevention and treatment of opportunistic infections in HIV-infected adults and adolescents: recommendations from the Centers for Disease Control and Prevention, the National Institutes of Health, and the HIV Medicine Association of the Infectious Diseases Society of America. AIDSinfo 2017:T-10 (http://aidsinfo.nih.gov/contentfiles/ lvguidelines/adult_oi.pdf).

DOI: 10.1056/NEJMc1709123

THE AUTHORS REPLY: Giovane et al. inquired whether the treatment effects differed on the basis of disease severity or preexisting medical conditions. First, we caution that criteria for severe disease have not been defined for talaromycosis. Second, international guidelines recommending itraconazole for mild to moderate disease and amphotericin B for severe disease are based solely on expert opinions and case series., ${ }^{1,2}$ In our analysis of mortality over 24 weeks in prespecified subgroups of patients, the observed benefit of amphotericin B over itraconazole was larger among patients who may have been susceptible to more severe infection (e.g., patients with newly diagnosed AIDS who had not started therapy for human immunodeficiency virus, patients with a history of injection drug use, patients with funge- mia, and patients with a CD4 cell count of $<50$ cells per cubic millimeter). However, statistical tests did not clearly establish heterogeneity of the treatment effect across subgroups (see Figs. S2 and S3 in the Supplementary Appendix of our article).

It is well established that adequate absorption of an itraconazole capsule depends on an acidic gastric environment and is highly variable depending on the relative timing of food intake and some coadministered drugs such as rifampicin and efavirenz. ${ }^{3,4}$ Our patients were instructed to take itraconazole immediately after a full meal or with an acidic beverage, and the administration was directly observed during hospitalization. We concur with Brüggemann et al. that therapeutic drug monitoring can ensure adequate itraconazole exposure. Unfortunately, therapeutic drug monitoring for antifungal drugs is unavailable in Vietnam and is unlikely to be established soon in countries in which talaromycosis is endemic and in which resources are limited. Our study was a pragmatic clinical trial that was designed to test the antifungal drugs and doses that are currently being used to treat talaromycosis and thereby inform the development of treatment guidelines in the region.

Thuy Le, M.D., D.Phil.

Guy Thwaites, M.D., Ph.D.

Marcel Wolbers, Ph.D.

\section{Oxford University Clinical Research Unit Ho Chi Minh City, Vietnam thuyl@oucru.org}

Since publication of their article, the authors report no further potential conflict of interest.

1. Kaplan JE, Benson C, Holmes KK, Brooks JT, Pau A, Masur H. Guidelines for prevention and treatment of opportunistic infections in HIV-infected adults and adolescents: recommendations from CDC, the National Institutes of Health, and the HIV Medicine Association of the Infectious Diseases Society of America. MMWR Recomm Rep 2009;58(RR-4):1-207.

2. Angus BJ, Schmid ML, Dockrell DH, Grant D. Travel-related opportunistic infections. In: British HIV Association and British Infection Association guidelines for the treatment of opportunistic infection in HIV-seropositive individuals 2011. HIV Med 2011;12:Suppl 2:88-101.

3. Lohitnavy $\mathrm{M}$, Lohitnavy $\mathrm{O}$, Thangkeattiyanon $\mathrm{O}$, Srichai W. Reduced oral itraconazole bioavailability by antacid suspension. J Clin Pharm Ther 2005;30:201-6.

4. Brüggemann RJ, Alffenaar JW, Blijlevens NM, et al. Clinical relevance of the pharmacokinetic interactions of azole antifungal drugs with other coadministered agents. Clin Infect Dis 2009;48:1441-58

DOI: $10.1056 /$ NEJMc1709123 